\title{
Susceptibilidad a Deslizamiento de Ladera mediante el Sistema de Información Geográfica
} Geographic Information System Landslide Susceptibility to
Landslides

\author{
Ruth Mariela Quispe-De La Cruz \\ Universidad Nacional de Huancavelica, Huancavelica, Perú \\ E-mail: ruthquisde@gmail.com ORCID: https://orcid.org/0000-0001-7260-4311 \\ Nilo Abelardo Enríquez-Nateros \\ Universidad Nacional de Huancavelica, Huancavelica, Perú \\ E-mail: niloeamb2@gmail.com ORCID: https://orcid.org/0000-0001-8233-6912 \\ Carlos Alberto Herrera-Quispe \\ Universidad Nacional de Huancavelica, Huancavelica, Perú \\ E-mail: carlosalbertoherrera15@gmail.com ORCID: https://orcid.org/0000-0003-2169-6214 \\ René Antonio Hinojosa-Benavides \\ Universidad Nacional Autónoma de Huanta, Perú \\ E-mail: rhinojosa@unah.edu.pe ORCID: https://orcid.org/0000-0002-0452-3162
}

\section{Resumen}

El deslizamiento de ladera es considerado uno de los fenómenos geológicos con mayor destrucción que impactan a la humanidad, llegando a causar grandes pérdidas humanas y materiales. El objetivo del presente trabajo de investigación bibliográfico fue demostrar la eficacia de la aplicación de un sistema de información geográfica (SIG) para el estudio de la susceptibilidad a deslizamiento de laderas a través de mapas susceptibles, para lo cual se desarrolló un análisis sistemático de artículos científicos publicados en revistas científicas como Engineering Geology, Investigaciones Geográficas, TecnoLógicas, Geomorphology, Terra, Revista Natural Hazards and Earth System Sciences, Revista de Estudios Latinoamericanos sobre Reducción del Riesgo de Desastres, entre otras, ubicadas en bases de datos como SciELO, World Wide Science, Scopus, ScienceDirect, Elsevier, entre las principales. Entre los resultados se tiene, que con la aplicación de un SIG se determina el porcentaje y grado de áreas que presentaran susceptibilidad a deslizamiento a ladera, en la cual se laboró con la metodología empleada por el SIG, por tanto, se concluye que la ejecución de mapas de susceptibilidad a deslizamiento de ladera debería ser de trascendencia debido a que serviría como soporte en la organización de un territorio, asegurando la estabilidad pública y un futuro sostenible. 
Palabras Clave

Mapas susceptibles, deslizamiento de ladera, SIG.

\section{Abstract}

The landslide is considered one of the most destructive geological phenomena that impact humanity, causing great human and material losses. The aim of this bibliographic research work was to demonstrate the effectiveness of the application of a geographic information system (GIS) for the study of slope landslide susceptibility through susceptibility maps, for which a systematic analysis of scientific articles published in scientific journals such as Engineering Geology, Investigaciones Geográficas, TecnoLógicas, Geomorphology, Geomorphology, Geomorphology and Geomorphology was developed, Investigaciones Geográficas, TecnoLógicas, Geomorphology, Terra, Revista Natural Hazards and Earth System Sciences, Revista de Estudios Latinoamericanos sobre Reducción del Riesgo de Desastres, among others, located in databases such as SciELO, World Wide Science, Scopus, ScienceDirect, Elsevier, among the main ones. Among the results is that with the application of a GIS it is determined the percentage and degree of areas that present susceptibility to landslide to slope, which worked with the methodology used by the GIS, therefore, it is concluded that the execution of maps of susceptibility to landslide of slope should be of transcendence because it would serve as support in the organization of a territory, ensuring public stability and a sustainable future.

\section{Keywords}

susceptible maps, landslide, slope, SIG.

\section{Resumo}

O deslizamento de terras é considerado um dos fenômenos geológicos mais destrutivos que afetam a humanidade, causando grandes perdas humanas e materiais. $\mathrm{O}$ objetivo do presente trabalho de pesquisa bibliográfica foi demonstrar a eficácia da aplicação de um sistema de informação geográfica (SIG) para o estudo da suscetibilidade ao deslizamento de taludes através de mapas de suscetibilidade, para o qual foi desenvolvida uma análise sistemática de artigos científicos publicados em revistas científicas como Engineering Geology, Investigaciones Geográficas, TecnoLógicas, Geomorphology, Geomorphology, Geomorphology e Geomorphology, Investigaciones Geográficas, TecnoLógicas, Geomorfologia, Terra, Revista Natural Hazards and Earth System Sciences, Revista de Estudios Latinoamericanos sobre Reducción del Riesgo de Desastres, entre outras, localizadas em bancos de dados como SciELO, World Wide Science, Scopus, ScienceDirect, Elsevier, entre as principais. Entre os resultados está que a aplicação de um SIG determina a porcentagem e o grau de áreas suscetíveis a deslizamentos de terra, nos quais se trabalhou com a metodologia utilizada pelo SIG, portanto, conclui-se que a execução de mapas de suscetibilidade a deslizamentos de terra deve ser importante porque serviria de apoio na organização de um território, garantindo a estabilidade pública e um futuro sustentável. 


\section{Palavras-chave}

Mapas suscetíveis, deslizamento de terras, SIG.

\section{Introducción}

El deslizamiento de laderas comprende el desplazamiento de suelo, rocas, escombros, descendiendo del talud a modo de caídos, deslizamientos y flujos. (CENAPRED, 2017), y es considerado como el segundo fenómeno geológico que provoca las mayores pérdidas humanas y materiales del mundo. Así mismo Aye et al. (2016) asegura que el deslizamiento de ladera ocurre naturalmente, sin embargo, esos deslizamientos también están involucrados con los hechos que el ser humano provoca al medio ambiente distorsionando de esta forma la morfología del territorio.

En vista a todo ello se establece de las áreas propensas a originar un deslizamiento de laderas, se conoce como análisis de susceptibilidad (GEMMA, 2007), considerada como la posibilidad espacial relativa de que suceda un deslizamiento de ladera en una cierta zona en base a las condiciones que tenga el terreno (Rossi et al., 2010a). se menciona que existen diversos métodos usados que nos permiten determinar a escalas territoriales la susceptibilidad a deslizamiento de laderas. Entre ellos son los métodos estadísticos bivariado y multivariado (Aristizábal et al., 2019). Según Gupta y Shukla (2018), es un desafío importante al preparar el mapa de susceptibilidad de deslizamientos de ladera para la estimación de la dimensión de los deslizamientos, para el cual se necesitan datos satelitales de alta resolución y la aplicación de un SIG.

El presente análisis ejecuta una revisión completa sobre la investigación de susceptibilidad a deslizamiento de ladera cuyo objetivo es que por medio de este análisis se posibilite la identificación de la susceptibilidad a deslizamiento de laderas, que es un camino necesario para la gestión de riesgo a desastres, ya que genera el conocimiento base para la toma de decisiones en referencia a ordenamiento territorial y la disminución del riesgo actual (Quesada et al., 2017), teniendo en consideración la estabilidad de la población. Pero todo ello tomando como base fundamental para dicho estudio el conocimiento del territorio a través del uso de un SIG. 
Del mismo modo, es necesario analizar por diferentes autores la posible solución al problema del deslizamiento de laderas y como se origina, ya que es un punto primordial para comprender y analizar; y así dar una posible solución apropiada (Rivera, 2020).

\section{Estado del arte o Marco Teórico}

\subsection{Susceptibilidad de deslizamiento de ladera}

2.1.1 Deslizamiento de ladera : Según Van Westen (2005) menciona que son movimiento de masa de tierra, roca o debris, pendiente abajo; de la misma manera Irasema (2008) explica que el deslizamiento es proveniente del término inglés "landslide" y que dicho deslizamiento ocurre a lo largo de una superficie de falla producto de una gran deformación. Los factores que favorecen al desarrollo del deslizamiento de ladera es la morfología del terreno, exceso o reducción de carga por obras humanas, cambio del ángulo de inclinación de una superficie, deforestación, agua subterránea, alternancia de humectación-sequedad, congelamiento-deshielo, movimientos tectónicos, actividad volcánica, entre otros (Quesada, 2018).

Los cambios de flujo y rumbo de los escurrimientos es producto de las variaciones topográficas y de uso de suelo (Doyle et al., 2000), provocando la disminución de los niveles de infiltración, ello siendo considerado como los detonantes para la generación de deslizamiento de ladera (Lepore et al., 2012), aumentando las probabilidades de ocurrencia, especialmente durante huracanes cuando se incremente la intensidad y duración de la precipitación (Murillo, 2002). La importancia de conocer el comportamiento espacial y temporal del fenómeno de deslizamiento de ladera (Paz et al., 2017) radica en las condiciones de hidrología, edafología, geología, uso del suelo (factores condicionantes), se suman los desencadenantes como las lluvias intensas y la sismicidad (Lugo et al., 2005).

La estabilidad de un talud se rige por factores que aportan fuerzas a lo largo del plano de deslizamiento. A escala de la ladera, la dinámica que condujo a un deslizamiento puede reconstruirse con relativa facilidad mediante estudios geotécnicos (Lu et al., 2017). Del mismo modo Arabameri et al. (2019b) menciona que los deslizamientos de tierra son un desastre natural común que ocurre en muchas zonas montañosas de todo el mundo afectando a la vida y a las propiedades de miles de personas cada año. Para reducir los 
daños causados por los deslizamientos de tierra, la predicción espacial de la susceptibilidad es uno de los enfoques más comunes (Arabameri et al., 2019c).

\subsubsection{Tipos de deslizamiento:}

\subsubsection{Deslizamientos Superficiales.}

Se refiere a la cantidad de movimiento que presenta un deslizamiento superficial, es de poquísimos centímetros por año, pero puede dañar una gran área de la superficie. Este deslizamiento puede ser causado por el cambio climático referidas al contenido de humedad de un suelo blando o alterado. Por otro lado, se afirma que el deslizamiento superficial presenta laderas empinadas muy amplias, pero no profundas, la gran mayoría de la superficie terrestre es utilizada para la agricultura, cultivos, pastoreo y una ganadería extensiva (De Matteis et al., 2003)

\subsubsection{Deslizamientos Rotacional.}

Para poder determinar un movimiento de masa de este tipo, se debe concluir que la curva originada sea porque su centro de giro es mayor al centro de gravedad del área de movimiento (De Matteis et al., 2003). Así mismo este deslizamiento son frecuentes en suelos homogéneos (cohesivos), se caracterizan por que la superficie de falla tiene forma curva o forma de cuchara, en este deslizamiento la masa deslizada se acumula al pie de la ladera, así como un lóbulo.

\subsubsection{Deslizamientos de Traslación.}

Con respecto a los deslizamientos traslacionales pueden generarse en suelos y rocas, la rotura se da a través de una o varias superficies de falla (estratificación, contacto entre rocas, planos de debilidad) que son planas y se orientan a favor del talud (ladera). Debido a las características de la superficie de falla, en los deslizamientos traslacionales la masa se desliza más rápido que en los deslizamientos rotacionales.

\subsubsection{Factores Causales del Deslizamiento de Laderas:}

\subsubsection{Factores Condicionantes.}

Este factor está determinado por los cambios morfológicos en la geometría de las laderas por procesos de urbanización, deforestación, explotación de materiales, 
condiciones hidrológicas, y aplicación de cargas estáticas o dinámicas al talud (CENEPRED, 2017)

Así mismo Zamora (2007) expresa que estos factores son los que siempre están presentes dentro del área, tales como la geomorfología local, hidrología, condiciones geológicas y vegetación. También es conocido en término de ocurrencia en lugar de condicionantes.

\subsubsection{Factores Desencadenantes.}

Actúan como fuerzas externas que interactúan con los factores condicionantes en el área de interés, produciendo un efecto complejo que podría afectar la inestabilidad y desencadenar un deslizamiento (González de Vallejo, 2002). Desde esta perspectiva, se puede explicar que los factores desencadenantes son situaciones que pueden desestabilizar una ladera tal como sismos, intensas lluvias y la intervención antrópica (Montiel et al., 2007)

Así mismo según Legorreta et al. (2013) en las zonas volcánicas por sus características geomórficas, tienen un gran potencial para generar deslizamientos y flujos de escombros en sus laderas debido a su elevado relieve, laderas escarpadas, debilitamiento progresivo del edificio volcánico por la alteración hidrotermal, acumulación de voluminosos depósitos de flujos piroclásticos y otras características geográficas.

2.1.4 Susceptibilidad a deslizamiento: La susceptibilidad tiene como propósito predecir la existencia de un fenómeno peligroso y analizar su potencial. Ante la situación de un deslizamiento de ladera, la amenaza se expresa a modo de mapas, y los territorios se catalogan según su tendencia a fracturarse más o menos (mapas de susceptibilidad y peligrosidad) (Corominas et al., 2014)

Por otro lado, GEMMA (2007) menciono que la identificación de las áreas propensas a fallas a deslizamiento de laderas se denomina análisis de susceptibilidad; para el estudio del grado de susceptibilidad a deslizamiento está definida por los factores inherentes al terreno, por la cual, en un deslizamiento de ladera, la clave es identificar el punto de falla 
inicial. La capacidad de expansión del deslizamiento es dependiente de la energía potencial, y las propiedades del recorrido.

De manera similar, la susceptibilidad a deslizamiento de ladera se puede tener en consideración como una función de distribución espacial, definida como la densidad esperada de la masa desplazada, que es el numero promedio de ocurrencias por km2 por año. En la cual se debe elaborar un mapa de susceptibilidad para cada tipo de deslizamiento de ladera debido a que por su naturaleza las amenazas pueden exponerse diferentes en cada situación (GEMMA, 2007).

2.1.5 Análisis de la susceptibilidad a Deslizamiento: Para este análisis se han planteado y probado diversos procedimientos y técnicas, pero aún no se ha llegado a un acuerdo general sobre los métodos más adaptados para evaluar los fenómenos. Una revisión de la literatura propuesta por Guzzetti et al. (1999) mostró una predilección por los enfoques indirectos y cuantitativos basados en modelos estadísticos (es decir, probabilísticos) o impulsados por procesos (es decir, geotécnicos).

Según Corominas et al. (2014), Guzzetti et al. (1999) y Rossi et al. (2010b). El método de análisis de susceptibilidad a deslizamientos basados en la física se basa en el modelado de los procesos de rotura de taludes. Los métodos solo son adecuados para áreas pequeñas con propiedades geológicas y geomorfológicas subjetivamente semejantes y tipos básicos de deslizamientos de ladera.

De manera similar, la susceptibilidad a deslizamiento de ladera se puede tener en consideración como una función de distribución espacial, definida como la densidad esperada de la masa desplazada, que es el numero promedio de ocurrencias por km2 por año. En la cual se debe elaborar un mapa de susceptibilidad para cada tipo de deslizamiento de ladera debido a que por su naturaleza las amenazas pueden exponerse diferentes en cada situación (GEMMA, 2007).

La predicción de la susceptibilidad a los desprendimientos muestra la probabilidad espacial de que se produzcan y es una tecnología clave en la supervisión, la alerta temprana y la evaluación de los deslizamientos de tierra (Wu et al., 2016). Cualquier modelo de susceptibilidad a los desprendimientos depende estrictamente de la unidad cartográfica 
que se elija (Rossi y Reichenbach, 2016). Una unidad cartográfica subdivide esencialmente cualquier área de estudio en objetos geográficos a los que el modelo asigna en última instancia las estimaciones de susceptibilidad. Entre las unidades cartográficas propuestas, las celdas de cuadrícula y las unidades de pendiente (SU) son las más comunes en la literatura (Reichenbach et al., 2018).

Los datos sobre la presencia de deslizamientos de tierra se suelen muestrear basándose en el inventario de deslizamientos de tierra, que a menudo se realiza mediante la interpretación de fotografías aéreas con comprobaciones sobre el terreno (Pham et al., 2018a; Pham et al., 2018b) o estudios de campo. Su calidad es relativamente alta (Süzen y Doyuran, 2004b; Yilmaz, 2010). Sin embargo, los datos de ausencia no están disponibles directamente y a menudo se aproximan a las condiciones de los lugares en los que no se han producido desprendimientos. Por lo tanto, la calidad o la fiabilidad de estos datos aproximados es a menudo desconocida (Zhu et al., 2014).

\subsection{Sistema de Información Geográfica}

2.2.1 ¿Que es un Sistema de Información Geográfica (SIG)?: El Sistema de Información Geográfica (SIG) es un grupo de programas, equipos, métodos, datos, perfectamente incluidos, que permiten recolectar, guardar, procesar y examinar dichos datos de alusión geográfica, de esta forma desde aplicaciones de generación de información derivada. El propósito de SIG es solucionar dificultades complejas de organización y gestión. Además, hace referencia al sistema de coordenadas terrestres que se conceptualiza como una sección del modelo de realidad y está elaborada para satisfacer necesidades concretas de información; permitiendo a los usuarios editar datos, mapas, examinar información espacial, producir consultas interactivas, y exponer los resultados de cada una de estas operaciones (López, 2013).

La ventaja principal de los SIG es que facilitan diferentes operaciones que se pueden realizar con las distintas coberturas generadas en base a mapas temáticos de una determinada zona, de esta forma se generan los mapas de susceptibilidad a la ocurrencia de deslizamientos (Cereceda, 2006) 
2.2.1 SIG como un modelo de la realidad: Los SIG pueden reproducir la realidad según un propósito específico, representando fenómenos con mayor o menor complejidad y según reglas definidas. En este sentido, el SIG se hace como un verdadero modelo de realidad, y como en cualquier modelo, es selectivo, pues constantemente aspira destacar los aspectos que interesan a los usuarios y descartar los irrelevantes.

\section{Materiales y métodos}

Se ejecutó una revisión bibliográfica en distintas bases de datos como SciELO, World

Wide Science, Scopus, ScienceDirect, Elsevier, empleando descriptores como "Mapas susceptibles", "deslizamiento de ladera", "SIG"; así mismo se desarrolló un estudio sistemático de artículos científicos publicados en revistas científicas como Engineering Geology, Investigaciones Geográficas, TecnoLógicas, Geomorphology, Terra, Revista Natural Hazards and Earth System Sciences, Revista de Estudios Latinoamericanos sobre Reducción del Riesgo de Desastres, entre otras, desarrollando el estado de arte en sus fases heurística y hermenéutica.

\section{Resultados}

Entre los resultados de la presente investigación bibliográfica se demuestra que mediante el uso del SIG se tiene como resultado el mapa de susceptibilidad a deslizamientos partiendo de la ejecución de una base de datos de distribución espacial a deslizamientos de ladera.

EI SIG se complementa con una herramienta valiosa y es el levantamiento geomorfológico que proporciona una información precisa y concreta de los procesos geomorfológicos, de las formas resultantes y los fenómenos naturales asociados, como es el caso de los deslizamientos de laderas. (Aceves et al., 2016)

Con base a la verificación de los resultados obtenidos, es posible garantizar la efectividad del sistema de información geográfico, donde muestra un buen manejo frente al análisis de susceptibilidad a deslizamiento de ladera.

El resultado al análisis de susceptibilidad fue a través de mapas propensos para cada tipo de deslizamiento, ya que por naturaleza el peligro puede representarse de diferente 
manera, los datos preliminares del modelo de sistema de información geográfico indican un mapa de peligro por deslizamiento de ladera, dichos resultados además se puede tomar como alusión para entender el mecanismo y la cinemática a los deslizamiento de ladera; por consiguiente la elaboración de un mapa de amenaza a deslizamiento de ladera puede crear información fundamental que posibilita a los causantes tomar elecciones primordiales en cuestiones de gestión de territorio.

\section{Discusión}

Gupta y Shukla (2018) argumentan que para la elaboración de mapas de susceptibilidad para la estimación del grado de deslizamiento de laderas es necesario tener datos satelitales de máxima resolución y la aplicación de un SIG, todo ello es corroborado por Quesada et al. (2017), donde indica que la base fundamental para el deslizamiento de ladera es el conocimiento del territorio a través del uso de un SIG cuyo objetivo es que por medio de este análisis se posibilite la identificación de la susceptibilidad a deslizamiento de laderas, siendo considerado un base necesario para la gestión de riesgo a desastres.

Así mismo Cereceda (2006) afirma que la ventaja principal del uso SIG es que facilitan diferentes operaciones que se pueden realizar con las distintas coberturas generadas en base a mapas temáticos de un determinado territorio, de esta forma se generan los mapas de susceptibilidad a la ocurrencia de deslizamientos de laderas.

\section{Conclusiones}

El sistema de modelación vía remota es una herramienta de gestión útil, los modelos basados en un SIG pueden responder rápidamente a una amplia gama de problemas abarcando a grandes escalas. El resultado funciona como un mapa base para detectar superficies que necesitan una atención mejorada por medio de una averiguación técnica específica.

Además, cabe decir que dichos resultados tienen la posibilidad de ser complementados con otra información adicional, como las propiedades socio económicas de las poblaciones vulnerables que posibilita el diseño y utilización de nuevos indicadores de peligro, vulnerabilidad social y valoraciones de base para el ordenamiento territorial. reclama. 


\section{Referencias}

Arabameri, A., Pradhan, B., Rezaei, K., Lee, S., Sohrabi, M. (2019b). An ensemble model for landslide susceptibility mapping in a forested area. Revista Geocarto International, 35(15), 1680-1705. https://n9.cl/osna6

Arabameri, A., Pradhan, B., Rezaei, K., Sohrabi, M., Kalantari, Z. (2019c). GIS-based landslide susceptibility mapping using numerical risk factor bivariate model and its ensemble with linear multivariate regression and boosted regression tree algorithms. Revista Journal of Mountain Science, 16, 595-618. https://n9.cl/z2qrk

Aceves, J., Gabriel, P., Lugo, J., Umaña, J., Legorreta, H. (2016). Sistemas de información geográfica y cartografía geomorfológica aplicados al inventario de deslizamientos y cartografía de susceptibilidad en la cuenca del río El Estado, Pico de Orizaba, México. Revista Investigaciones Geográficas, 91, 43-55. https://doi.org/10.14350/rig.46503

Aristizábal, E., Vasquez, M., y Ruíz, D. (2019). The Susceptibility of Landslide Evaluation and Zoning by Statistical Methods. Revista TecnoLógicas, 22 (46), 39-60. https://doi.org/10.22430/22565337.1247

Aye, Z. C., Jaboyedoff, M., Derron, M. H., Van Westen, C. J., Hussin, H. Y., Ciurean, R. L., Frigerio, S., y Pasuto, A. (2016). An Interactive Web-GIS Tool for Risk Analysis: A Case Study in the Fella River Basin, Italy. Natural Hazards and Earth System Sciences. Revista Natural Hazards and Earth System Sciences, 16, 85-101. https://n9.cl/w8bgb CENAPRED, Centro Nacional de Prevención de Desastres (2017). Conceptos Básicos y Factores Que Rigen La Estabilidad En Laderas y Taludes. México, Centro Nacional de Prevención de Desastres

Cereceda, I. (2006). Uso de los SIG en el análisis de susceptibilidad y predicción de deslizamientos. Compendio de Trabajos de Investigación CNDG - Biblioteca Instituto Geofísico del Perú, 7, 191 - 202. https://n9.cl/vdwfl

Corominas, J., Van Westen, C., Frattini, P., Cascini, L., Malet, J.-P., Fotopoulou, S., Catani, F., Van Den Eeckhaut, M., Mavrouli, O., Agliardi, F., Pitilakis, K., Winter, M. G., Pastor M., Ferlisi, S., Tofani, V., Hervás, J. y Smith, J. T. (2014). Recommendations for the quantitative analysis of landslide risk. Bulletin of Engineering Geology and the Environment, 73, 209-263. https://doi.org/10.1007/s10064-013-0538-8

De Matteis, A., Angelone, S. y Garibay, M. (2003). Geología y Geotecnia - Estabilidad de taludes, Universidad Nacional de Rosario, Argentina.

Doyle, M., Harbor, J., Rich, C. y Spacie, A. (2000). Examining the effects of urbanization on streams using indicators of geomorphic stability. Revista Physical Geograpfhy, 21(2), 155-181. https://doi.org/10.1080/02723646.2000.10642704

GEMMA, Grupo de Estándares para Movimientos en Masa (2007). Movimientos en Masa en la Región Andina: Una guía para la evaluación de amenazas. Proyecto 
Multinacional Andino: Geociencias para las Comunidades Andinas. Publicación geológica multinacional, 4, 0717-3733.

Gupta, S. y Shukla, D. (2018). Application of drone for landslide mapping, dimension estimation and its 3D reconstruction. Journal of the Indian Society of Remote Sensing, 46, 903-914. https://doi.org/10.1007/s12524-017-0727-1

Guzzetti, F., Carrara, A., Cardinali, M., y Reichenbach, P. (1999). Landslide hazard evaluation: A review of current techniques and their application in a multi-scale study, central Italy. Revista Geomorphology, 31 (1-4), 181-216. https://n9.cl/7s3ml

Irasema Alcántara Ayala, A. E. (2008). Inestabilidad de Laderas. Serie Fascículos, 39.

Montiel, K., Gouveia, M., y Montes, E. (2007). Influencia de la intervención antrópica en la ocurrencia de procesos de ladera. Microcuenca de la Quebrada Ramos, flanco Norandino venezolano. Revista Terra, 23(34), 35-69. https://n9.cl/drj0i

Murillo-Sánchez, M. E. (2002). Estudio del efecto del cambio de uso de suelo en el escurrimiento en la subcuenca 24Bf "Monterrey", aplicando un sistema de información geográfica, [Tesis doctoral, Instituto Tecnológico y de Estudios Superiores de Monterrey]. E-Archivo. http://hdl.handle.net/11285/568284.

Legorreta, P., M. Bursik, Ramírez, M. Contreras, T. Polenz, M. Lugo, J. Paredes y Arana, L. (2013). Landslide inventory mapping and landslide susceptibility modeling assessment on the SW flank of Pico de Orizaba volcano, Puebla-Veracruz, Mexico. Revista Zeitschrift für Geomorphologie, 57(3), 371-385. http://dx.doi.org/ 10.1127/0372-8854/2012/0097

Lepore, C., Kamal, S.A., Shanahan, P. y Bras, R.L. (2012). Rainfall-induced landslide susceptibility zonation of Puerto Rico. Revista Environmental Earth Sciences, 66, 16671681. http://dx.doi.org/ 10.1007/s12665-011-0976-1

Lu, Q., Liu, Y., Yang, Q. (2017). Stability analysis of earthquake-induced rock slope based on back analysis of shear strength parameters of rock mass. Revista Engineering Geology, 228, 39-49. https://doi.org/10.1016/j.enggeo.2017.07.007

Lugo, J., Zamorano, J., Capra, L., Inbar, M. y Alcántara, I. (2005). Los procesos de remoción en masa en la Sierra Norte de Puebla, octubre de 1999: Causas y efectos. Revista Mexicana de Ciencias Geológicas, 22(2), 212-228. https://n9.cl/bg2cr

Paz, J., González, R., Gómez, M. y Velasco, J. (2017). Metodología para elaborar mapas de susceptibilidad a procesos de remoción en masa, análisis del caso ladera sur de Tuxtla Gutiérrez, Chiapas. Revista Investigaciones Geográficas, 92. http://dx.doi.org/10.14350/rig.52822

Quesada, A. (2018). Comparación de la Metodología Mora-Vahrson y el Método Morfométrico para Determinar Áreas Susceptibles a Deslizamientos en la Microcuenca del Río Macho, Costa Rica. Revista Geográfica de América Central, 61,1745. http://dx.doi.org/10.15359/rgac.61-2.1 
Quesada, A. y Barrantes, G. (2017). Modelo morfométrico para determinar áreas susceptibles a procesos de ladera. Revista Investigaciones Geográficas, 94. http://dx.doi.org/10.14350/rig.57318

Pham, B., Son, L., Hoang, T., Nguyen, D., Tien Bui, D. (2018a). Prediction of shear strength of soft soil using machine learning methods. Revista CATENA, 166, 181-191. https://doi.org/10.1016/j.catena.2018.04.004

Pham, B., Tien Bui, D., Prakash, I. (2018b). Bagging based support vector machines for spatial prediction of landslides. Revista Environmental Earth Sciences. 77, 146. https://n9.cl/3at52

Reichenbach, P., Rossi, M., Malamud, B.D., Mihir, M., Guzzetti, F. (2018). A review of statistically-based landslide susceptibility models. Revista Earth-Science Reviews, 180, 60-91. https://doi.org/10.1016/j.earscirev.2018.03.001

Rivera, O. (2020). Fotogrametría de Drones para la Prevención de Deslizamientos de Tierra en la Ciudad de México. Revista de Estudios Latinoamericanos sobre Reducción del Riesgo de Desastres REDER, 4(2), 85-96. https://n9.cl/12nj

Rossi, M. y Reichenbach, P. (2016). LAND-SE: a software for statistically based landslide susceptibility zonation, version 1.0. Revista Geoscientific Model Development, 9 (10), 3533-3543. https://doi.org/10.5194/gmd-9-3533-2016

Rossi, M., Witt, A., Guzzetti, F., Malamud, B.D., y Peruccacci, S. (2010b). Analysis of historical landslide time series in the Emilia-Romagna region, northern Italy. Revista Earth Surface Processes and Landforms, 35 (10), 1123-1137. https://n9.cl/nntq

Rossi, M., Guzzetti, F., Reichenbach, P., Mondini, A., y Peruccacci, S. (2010a). Optimal landslide susceptibility zonation based on multiple forecasts. Revista Geomorphology, 114 (3), 129-142. https://n9.cl/cmdt

Süzen, M. y Doyuran, V. (2004b). Data driven bivariate landslide susceptibility assessment using geographical information systems: a method and application to Asarsuyu catchment, Turkey. Revista Engineering Geology, 71, 303-321. https://n9.cl/q4vtz

Van Westen, C. (2005). Introducción a los deslizamientos. Tipos y causas. International Institute for Geo- Information Science Earth Observation (ITC) de Holanda.

Wu, X., Shen, S., Niu, R. (2016). Landslide Susceptibility Prediction Using GIS and PSOSVM. Revista Geomatics \& Information Science of Wuhan University, 41 (5), 665-671. DOI:10.13203/j.whugis20130566

Yilmaz, I. (2010). The effect of the sampling strategies on the landslide susceptibility mapping by conditional probability and artificial neural networks. Revista Environmental Earth Sciences, 60, 505-519. https://n9.cl/ocq7y

Zhu, A.X., Wang, R., Qiao, J., Qin, C.Z., Chen, Y., Liu, J., Du, F., Yang, L., Zhu, T. (2014). An expert knowledge-based approach to landslide susceptibility mapping using GIS and fuzzy logic. Revista Geomorphology, 214, 128-138. https://n9.cl/btsab 